\title{
Affine Symmetry Tensors in Minkowski Space
}

\author{
Isaac Ahern*, Sam Cook \\ Department of Mathematics, University of Alaska, Anchorage, AK
}

Student: iahern@alaska.edu*

Mentor:scook@alaska.edu

\begin{abstract}
Killing vectors are generators of symmetries in a spacetime. This article defines certain generalizations of Killing vectors, called affine symmetry tensors, or simply affine tensors. While the affine vectors of the Minkowski spacetime are well known, and partial results for valence $n=2$ have been discussed, affine tensors of valence $n>2$ have never been exhibited. In this article, we discuss a computational algorithm to compute affine tensors in Minkowski spacetime, and discuss the results for affine tensors of valence $2 \leq n \leq 7$. After comparison with analogous results concerning Killing tensors, we make several conjectures about the spaces of affine tensors in Minkowski spacetime.
\end{abstract}

\section{KEYWORDS}

Affine Symmetry Tensors; Affine Vectors; Killing Tensors; Killing Vectors; Minkowski Spacetime; Dimension; Maple CAS; Lie Derivative; Generalized Killing Tensor

\section{INTRODUCTION}

The study of Killing tensors is the study of generalizations of certain vector fields, called Killing vectors, along algebraic lines. This paper examines tensors occurring on Minkowski space, the space of special relativity. There are 10 fundamentally different geometrical transformations on Minkowski space, called isometries, each with an associated vector field. These are known as Killing vectors of the space, which can be expressed as solutions of a certain equation, Killing's equation. The motivation for the study of affine symmetry tensors comes from examining the solutions to a more general equation than the Killing tensor equation; these generalizations will be examined in more detail in the next section. Essentially, the Killing tensors and the affine vectors of Minkowski space can be characterized as solutions of certain equations similar to the Killing equation, and by generalizing the Killing equation in an appropriate way, we may permit entirely new forms of solutions.

One way to generalize is to seek solutions to the following equation,

$$
\partial_{\left(\alpha_{1}\right.} \partial_{\alpha_{2}} \cdots \partial_{\alpha_{s}} \mathbf{X}_{\left.\alpha_{s+1} \cdots \alpha_{s+n}\right)}=0
$$

whose solutions are called Killing tensors of valence $n$ and order $s,{ }^{1}$ or sometimes generalized Killing tensors. ${ }^{2}$ All of the tensors mentioned above are generalized Killing tensors. The affine symmetry tensors studied in this paper are also a subclass of generalized Killing tensors. In a paper the authors became aware of after this paper was originally submitted, these were called homogeneous generalized Killing tensors in the work of Caviglia, et. al., ${ }^{2}$ where their relationship to the equation of geodesic deviation was studied. More recently, these tensors were studied as a direct generalization of affine vectors. ${ }^{3}$

The goal of the present work is to characterize the number of affine symmetry tensors of a given valence in the Minkowski spacetime. We accomplish this by computation using the software package Maple, for tensors of valence $n, 2 \leq n \leq 7$, which extends what is currently known for these tensors. The code for the valence 4 computation is attached to this article, and illustrative snippets are given in the text below. The code itself is also discussed in a later section, as are ways the code could be modified for similar computations in different spacetimes.

\section{PRELIMINARIES}

Tensors in $\mathbb{M}^{4}$. The Minkowski spacetime $\mathbb{M}^{4}=\left(\mathbb{R}^{4}, \eta\right)$, is the four dimensional flat spacetime of special relativity. The metric tensor $\eta$ on $\mathbb{M}^{4}$ is a valence 2 covariant tensor with components defined by the line element

$$
\mathrm{d} s^{2}=-\left(\mathrm{d} x^{0}\right)^{2}+\left(\mathrm{d} x^{1}\right)^{2}+\left(\mathrm{d} x^{2}\right)^{2}+\left(\mathrm{d} x^{3}\right)^{2} .
$$

We have $\eta=\eta_{a b} \mathrm{~d} x^{a} \mathrm{~d} x^{b}$, where we have used the Einstein summation convention indicating summation over repeated indices. Comparing with the line element given above, we see the components of the metric $\eta$ are $-\eta_{00}=1=\eta_{11}=\eta_{22}=\eta_{33}$, with all others zero. A 4 -vector is a valence 1 contravariant tensor, $\mathbf{X}$, which has components $\mathbf{X}^{\alpha}$, with the index $\alpha$ taking on the values $0,1,2,3$. We can relate a 4 -vector $\mathbf{X}^{\alpha}$ to its dual, the covector, or valence 1 covariant tensor, $\mathbf{X}_{\alpha}$ by

$$
\mathbf{X}_{\alpha}=\eta_{\alpha \beta} \mathbf{X}^{\beta} \text {. }
$$


This is another instance of the Einstein summation. That is, $\mathbf{X}_{\alpha}=\eta_{\alpha \beta} \mathbf{X}^{\beta}=\sum_{\beta=0}^{3} \eta_{\alpha \beta} \mathbf{X}^{\beta}$

A valence $n$ covariant tensor is a multilinear mapping taking $n$ tangent vectors as arguments. A symmetric valence $n$ tensor is one that is invariant under any permutation of its indices; we signify this by $\mathbf{X}_{\alpha_{1} \cdots \alpha_{n}}=\mathbf{X}_{\left(\alpha_{1} \cdots \alpha_{n}\right)}$. Then for any valence $n$ tensor $\mathbf{X}_{\alpha_{1} \cdots \alpha_{n}}$, we can define a corresponding symmetric tensor $\mathbf{X}_{\left(\alpha_{1} \cdots \alpha_{n}\right)}$, the symmetrization of $\mathbf{X}_{\alpha_{1} \cdots \alpha_{n}}$, in the following manner:

$$
\mathbf{X}_{\left(\alpha_{1} \cdots \alpha_{n}\right)}=\frac{1}{n !} \sum_{\sigma \in S_{n}} \mathbf{X}_{\sigma\left(\alpha_{1} \cdots \alpha_{n}\right)}
$$

that is, where the sum is taken over permutations $\sigma$ from the symmetric group $S_{n}$. The Lie derivative of a vector $\mathbf{X}$ in a direction $\mathbf{Y}, \mathcal{L}_{\mathbf{Y}} \mathbf{X}$, can be computed as the Lie bracket, or commutator $[\mathbf{X}, \mathbf{Y}]$ of $\mathbf{X}$ and $\mathbf{Y}$, given by

$$
\mathcal{L}_{\mathbf{Y}} \mathbf{X}:=[\mathbf{Y}, \mathbf{X}]=\mathbf{Y X}-\mathbf{X Y} \text {. }
$$

More precisely, for any smooth function $f$ on $\mathbb{M}^{4}$,

$$
[\mathbf{Y}, \mathbf{X}](f)=[\mathbf{Y}[\mathbf{X}]-\mathbf{X}[\mathbf{Y}]](f)=\mathbf{Y}(\mathbf{X}(f))-\mathbf{X}(\mathbf{Y}(f)) .
$$

Geometrically, the Lie derivative $\mathcal{L}$ is one measure of how vectors, or more generally tensors, on a manifold change.

The isometries $\widetilde{\boldsymbol{x}}$ on $\mathbb{M}^{4}$ are mappings that preserve the metric $\eta$; expressing this via the Lie derivative, we have that $\mathcal{L}_{\mathbf{X}} \eta=0$, where the vector $\mathbf{X}$ is tangent to the isometry $\widetilde{x}$. If we write out the components of this equation, we see it is equivalent to Killing's equation (after Wilhelm Killing) ${ }^{4}$ in $\mathbb{M}^{4}$

$$
\partial_{(\alpha} \mathbf{X}_{\beta)}=0,
$$

where $\partial_{\alpha}=\frac{\partial}{\partial x^{\alpha}}$ is the derivative with respect to the coordinate $x^{\alpha}$.

Generalizations. The solutions of Killing's equation are called the Killing vectors of the space. By analogy, we can generalize Killing's equation, and define a Killing tensor of valence $n$ to be any valence $n$ symmetric tensor $K_{\alpha_{1} \cdots \alpha_{n}}$ satisfying the equation

$$
\partial_{\left(\alpha_{n+1}\right.} K_{\left.\alpha_{1} \cdots \alpha_{n}\right)}=0 .
$$

It is apparent that the number of equations in the system for the Killing tensor grow quickly; counting, we can see that in the system for a Killing tensor of valence $n$ there are exactly

$$
\left(\begin{array}{c}
4+(n+1)-1 \\
(n+1)
\end{array}\right)=\frac{(n+4) !}{(n+1) ! 3 !}
$$

equations. Another generalization of the notion of Killing vectors is to consider the vectors satisfying

$$
\partial_{\alpha} \partial_{(\beta} \mathbf{X}_{\gamma)}=0
$$

These are called affine vectors. It is easy to see that a Killing vector is also an affine vector, since, if $\mathbf{X}$ is Killing, $\partial_{\alpha} \partial_{(\beta} \mathbf{X}_{\gamma)}=\partial_{\alpha} 0=0$. Hence, the space of affine vectors for a spacetime is at least as large as the space of Killing vectors. Geometrically, affine vectors generate affine motions, which are maps that take geodesic curves to geodesic curves while preserving the affine parameter.

A further generalization extending both of the previous notions is that given by the idea of what will be referred to here as an affine symmetry tensor, or more simply as an affine tensor, which is a symmetric valence $n$ tensor satisfying

$$
\partial_{\alpha_{1}} \partial_{\left(\alpha_{2}\right.} \mathbf{X}_{\left.\alpha_{3} \cdots \alpha_{n+2}\right)}=0
$$

As is the case with the Killing vectors, we see that a Killing tensor is also an affine tensor.

We denote the space of Killing tensors of valence $n$ over $\mathbb{M}^{4}$ by $\mathcal{K}_{n}\left(\mathbb{M}^{4}\right)$, and the space of affine tensors of valence $n$ over $\mathbb{M}^{4}$ by $\mathcal{A}_{n}\left(\mathbb{M}^{4}\right)$; as the context of working with $\mathbb{M}^{4}$ is clear, we shall also make use of the abbreviations $\mathcal{K}_{n}$ and $\mathcal{A}_{n}$. By previous observations then, we have that for any $n>0, \mathcal{K}_{n} \subset \mathcal{A}_{n}$.

We will give a short summary of some known results in the following section. Following this, we discuss the algorithm used to enumerate the affine tensors, and discuss the conclusions and conjectures we drew from the algorithmic output.

\section{SUMMARY OF KNOWN RESULTS}

Size of $\mathcal{K}_{n}$. Vector spaces of Killing tensors in spaces of constant curvature have been studied independently by Tacheuchi, ${ }^{5}$ Delong, ${ }^{6}$ and Thompson. ${ }^{7}$ We will follow the conventions of Thompson; he showed the dimension of the vector space consisting of valence $n$ Killing tensors in $\mathbb{M}^{4}$ is

$$
\left|\mathcal{K}_{n}\right|=\frac{(3+n) !(4+n) !}{3 ! 4 ! n !(n+1) !}=\frac{1}{4}\left(\begin{array}{c}
n+3 \\
n
\end{array}\right) \times\left(\begin{array}{c}
n+4 \\
n+1
\end{array}\right)
$$


This is the maximum number of Killing tensors in any 4-dimensional spacetime. In fact, if $K_{n}^{m}$ is the dimension of the vector space of Killing tensors, where $m$ is the dimension of the ambient space $\left(\mathrm{M}^{4}\right)$ and $n$ is the valence of the tensor, then

$$
K_{n}^{m} \leq \frac{(m+n-1) !(m+n) !}{(m-1) ! m ! n !(n+1) !}=\frac{1}{m}\left(\begin{array}{c}
n+m \\
n+1
\end{array}\right) \times\left(\begin{array}{c}
n+m-1 \\
n
\end{array}\right),
$$

and equality is achieved in spaces of constant curvature.

Many papers explicitly calculating the Killing tensors of a given valence in a particular spacetime have been published..$^{7,8,9,10}$ Moreover, the problems of finding the dimension of the vector space of generalized and generalized conformal Killing tensors have been considered by Nikitin and Prilpko ${ }^{1}$ and Eastwood. ${ }^{11,12}$ In this paper, we present a Maple algorithm to compute the affine tensors (and Killing tensors) in $\mathbb{M}^{4}$ for $n=1, \ldots, 7$; an example of this algorithm that calculates the valence $n=4$ tensors is included in the appendix of this paper. We include in this paper the values of $\left|\mathcal{K}_{n}\right|$ and $\left|\mathcal{A}_{n}\right|$ for each valence.

\section{AFFINE TENSORS IN $\mathbb{M}^{4}$}

The algorithm. A brief explanation of the algorithm is given below, which accompanies the attached sample code for the valence 4 affine symmetry tensors given in the appendix. The code is divided into 5 sections. We note that for space considerations, the output of the commands has been suppressed (by use of a colon instead of a semicolon). We have included small snippets of the output below for purposes of illustration.

In section 1 , we load the necessary packages for the computations, and define the spacetime $\mathbb{M}^{4}$ (by its metric $g=\eta$ ) within which we will work. We define the coordinates of the spacetime, and using these and the metric, compute the Christoffel symbols for computation in section 3. It is worth pointing out that the worksheet attached in the appendix can be used as a template for further calculations, involving tensors of other valences (i.e., the case $n \neq 4$ by very slightly modifying the code described below) or in different spacetimes, by modifying the initial data in this section of the code.

In section 2, we construct a generic valence 4 symmetric tensor, whose components we will be concerned with finding. The attached Maple worksheet includes the construction of a valence 4 covariant tensor; however it is possible to generalize the approach in an appropriate manner to construct a tensor of any valence.

In section 3, we build the system(s) of PDE which would like to solve. The Killing tensor equation is built from the symmetrization of the covariant derivative of the tensor. That is the purpose of the first two lines of this section. The ADK command then covariantly differentiates this, which produces the affine tensor equation. The KTSYS and ADESYS commands then tell Maple to explicitly form the corresponding systems of differential equations from these.

Figure 1. First few components of Affine System PDE.

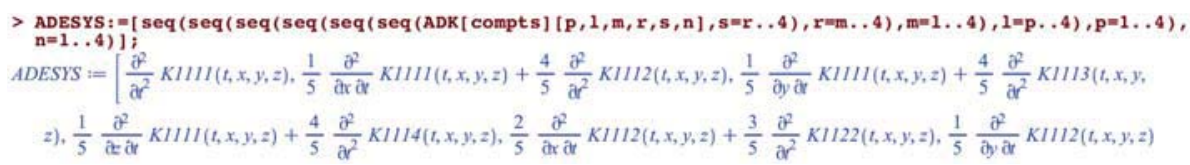

In section 4, we solve the system ADESYS to find the valence 4 (in this case) affine symmetry tensors. This is done using the pdsolve command. The pdetest command is used to verify that the given solutions actually solve the system ADESYS.

Figure 2. 1111 component of general solution, with arbitrary constants shown.

$$
\begin{aligned}
& \text { > so1: =pdsolve (ADESYS) ; } \\
& \text { sol }:=\left\{K I I I I(t, x, y, z)=\_C l t+\frac{1}{24}-C 2 x^{4}+\frac{1}{24}\left(4 \_C 3 y+4 \_C 4 z+4 \_C 5\right) x^{3}+\frac{1}{24}\left(6 \_C 6 y^{2}+\left(12 \_C 7 z+12 \_C 8\right) y+6 \_C 9 z^{2}\right.\right. \\
& \left.+12 \_C l O z+12 \_C l 1\right) x^{2}+\frac{1}{24}\left(4 \_C l 2 y^{3}+\left(12 \_C l 3 z+12 \_C l 4\right) y^{2}+\left(12 \_C l 5 z^{2}+24 \_C l 6 z+24 \_C l 7\right) y+4{ }_{-} C l 8 z^{3}\right. \\
& \left.+12 \_C 19 z^{2}+24 \_C 20 z+24 \_C 2 l\right) x+\frac{1}{24}-C 22 y^{4}+\frac{1}{24}\left(4 \_C 23 z+4-C 24\right) y^{3}+\frac{1}{24}\left(6 \_C 25 z^{2}+12 \_C 26 z+12 \_C 27\right) y^{2} \\
& +\frac{1}{24}\left(4-C 28 z^{3}+12 \_C 29 z^{2}+24-C 30 z+24 \_C 31\right) y+\frac{1}{24}-C 32 z^{4}+\frac{1}{6}-C 33 z^{3}+\frac{1}{2}-C 34 z^{2}+C 35 z+C C 36, K 1112(t, x, y, z)
\end{aligned}
$$

In section 5, we isolate the components of each solution. Each arbitrary constant in the solution, of the form _C $\mathrm{i}$, corresponds to a different independent solution of the system. So, for each arbitrary constant in the solution, we list here the non-zero components. The commands in this section are sensitive to the number of elements in the lists. Thus, the number of components and the number of solutions must be known for this section. The number of components can be computed. The number of solutions can be found with little effort by using Maple's built in search feature to search the output and determine the maximum index of the coefficients of the solutions, from the output of section 4 . The output can be made visible and searchable by changing the colon to a semicolon in the sol command in section 4 . 
Figure 3. Non-zero components of solution corresponding to C2.

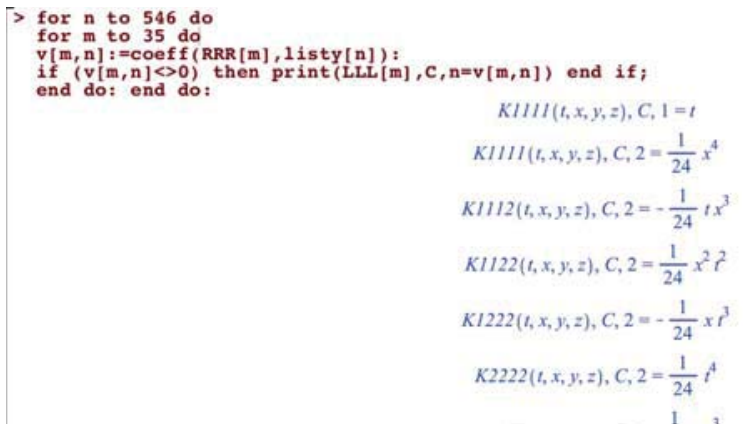

The number of parameters used to describe the solutions, or dimension of the corresponding solution space, was recorded and used to conjecture the dimension, $\left|\mathcal{A}_{n}\right|$, of $\mathcal{A}_{n}$; the data is recorded in the next section (see Table 1).

Results. Information about the number of equations and parameters is collected below:

Table 1. Parameter and Equation Sizes.

\begin{tabular}{|c|c|c|c|}
\hline valence $\mathbf{n}$ & \# Killing Tensor parameters & \# Affine Tensor parameters & \# Affine \& not Killing \\
\hline 1 & 10 & 20 & 10 \\
\hline 2 & 50 & 70 & 20 \\
\hline 3 & 175 & 210 & 35 \\
\hline 4 & 490 & 546 & 56 \\
\hline 5 & 1176 & 1260 & 84 \\
\hline 6 & 2520 & 2640 & 120 \\
\hline 7 & 4950 & 5115 & 165 \\
\hline
\end{tabular}

It appears from the data that the number of Killing tensors of order $n,\left|\mathcal{K}_{n}\right|$, for $n=1, \ldots, 7$ follows the OEIS sequence A006542, ${ }^{13}$ which matches the formula given by Thompson, and that the number of non-Killing affine tensors follows the Tetrahedral number sequence $T_{n}$, which is the OEIS sequence A000292. ${ }^{14}$

Based on the data collected for the affine tensors with valence $n=1, \ldots, 7$, we make the following observation and conjectures: 1. Our data for $\left|\mathcal{K}_{n}\right|$ indicates that

$$
\left|\mathcal{K}_{n}\left(\mathbb{M}^{4}\right)\right|=\frac{1}{4}\left(\begin{array}{l}
n \\
3
\end{array}\right) \times\left(\begin{array}{c}
n-1 \\
3
\end{array}\right)
$$

for $n=4,5,6, \ldots$ It is known that $\left|\mathcal{K}_{n}\right|=\frac{1}{4}\left(\begin{array}{c}n+4 \\ n+1\end{array}\right) \times\left(\begin{array}{c}n+3 \\ n\end{array}\right)$. These two forms are equivalent however, as can be seen by letting. $n \rightarrow n+m=n+4$ where $m$ is the dimension of $\mathbb{M}^{4}$. Then,

$$
\frac{1}{4}\left(\begin{array}{l}
n \\
3
\end{array}\right) \times\left(\begin{array}{c}
n-1 \\
3
\end{array}\right) \rightarrow \frac{1}{4}\left(\begin{array}{c}
n+4 \\
n+1
\end{array}\right) \times\left(\begin{array}{c}
n+3 \\
n
\end{array}\right)
$$

and hence $\left\{\left|\mathcal{K}_{n}\left(\mathbb{M}^{4}\right)\right|\right\}_{n \in \mathbb{N}, n \geq 4}=\left\{\frac{1}{4}\left(\begin{array}{c}n+4 \\ n+1\end{array}\right) \times\left(\begin{array}{c}n+3 \\ n\end{array}\right)\right\}_{n \in \mathbb{N}^{*}}$.

2. It appears that

$$
\left|\mathcal{A}_{n} \backslash \mathcal{K}_{n}\right|=\left(\begin{array}{c}
n+4 \\
3
\end{array}\right)=\left(\begin{array}{c}
(n+2)+2 \\
3
\end{array}\right) .
$$

$\left(\begin{array}{c}(n+2)+2 \\ 3\end{array}\right)=T_{n+2}$ also characterizes the Tetrahedral number sequence $T_{n},{ }^{14}$ for $n=1,2, \ldots$ but shifted forward two terms in the sequence. Observations 1. and 2. allow us to make the following conjecture as to the size of $\mathcal{A}_{n}$ :

$$
\left|\mathcal{A}_{n}\right|=\left|\mathcal{A}_{n} \backslash \mathcal{K}_{n}\right|+\left|\mathcal{K}_{n}\right|=\left(\begin{array}{c}
n+4 \\
3
\end{array}\right)\left[1+\frac{1}{4} \times\left(\begin{array}{c}
n+3 \\
3
\end{array}\right)\right] .
$$

In the computations for each $\mathcal{A}_{n}$, we also observe that the components of each tensor for $n=1, \ldots, 7$ are in the form of multivariable polynomials with degree less than or equal to $n$. We conjecture that this is the general form of the tensors in $\mathcal{A}_{n}$, that is, 
the components of each such tensor are multivariable polynomials with degree $\leq n$.

Finally, we conjecture that the number of affine tensors in a space follow a similar inequality to the form that Thompson found that is, that the affine symmetry tensors of valence $n$ for a manifold $\mathcal{M}$ of dimension $m$ are of the order $\mathcal{A}_{n}^{m}$ where

$$
\mathcal{A}_{n}^{m} \leq\left(\begin{array}{c}
n+m \\
m-1
\end{array}\right)\left[1+\frac{1}{m} \times\left(\begin{array}{c}
n+m-1 \\
m-1
\end{array}\right)\right]=\left(\begin{array}{c}
n+m \\
n+1
\end{array}\right)\left[1+\frac{1}{m} \times\left(\begin{array}{c}
n+m-1 \\
n
\end{array}\right)\right],
$$

with the inequality similarly achieving equality in spaces $\mathcal{M}$ of constant curvature, such as $\mathbb{M}^{4}$.

\section{REFERENCES}

1. Nikitin, A. G. and Prilpko, A., (1990) Generalized Killing Tensors and Symmetry of Klein-Gordon-Fock Equations, preprint 90.23, Acad. Sci Ukr SSR, Institute of Mathematics, Kiev, 1 - 59 (Preprint in English available at arxiv.org/pdf/mathph/0506002v1.pdf)

2. Caviglia, G., Salmistraro, F., and Zordan, C., (1982) Geodesic deviation and first integrals of motion, J. Math. Phys., 23, 2346 $-2352$

3. Cook, S. and Dray, T., (2009) Tensor generalizations of affine symmetry vectors, J. Math. Phys., 50, 122506 - 122512.

4. Martin, D., (2002) Manifold Theory: an Introduction for Mathematical Physicists (Horwood), 228 - 238, Simon \& Schuster, Sussex.

5. Tacheuchi, M., (1983) Killing tensor fields on spaces of constant curvature, Tsukuba J. Math., 7, $233-55$

6. Delong, R.P., (1982) Killing tensors and the Hamilton-Jacobi equation, PhD thesis, University of Minnesota

7. Thompson, G., (1986) Killing tensors in spaces of constant curvature, J. Math. Phys., 27, 2693 - 2699.

8. Caviglia, G. and Zordon, C., (1982) Third-Order Killing Tensors in the Schwarzschild spacetime, Gen. Relat. Gravit., 14, 27 30.

9. Ikeda, M. and Kimura, M., (1972) On Quadratic First Integrals in the General-Relativistic Kepler Problem, Tensor N. S., 25, $395-404$.

10. Walker, M. and Penrose, R., (1970) On Quadratic First Integrals of the Geodesic Equations for Type \{22\} Spacetimes, Comm. Math. Phys., 18, $265-274$.

11. Eastwood, M., (2005) Higher symmetry of the Laplacian, Annals of Mathematics, 161, 1645 - 1665

12. Eastwood, M., (2005) Representations via overdetermined systems, Contemp. Math., AMS, 368, 201- 210

13. The On-Line Encyclopedia of Integer Sequences, https://oeis.ong/A006542 (accessed Mar 2016)

14. The On-Line Encyclopedia of Integer Sequences, https://oeis.org/A000292 (accessed Mar 2016)

\section{ABOUT THE STUDENT AUTHOR}

Isaac Ahern is a recent graduate of the University of Alaska, Anchorage, where this research was completed. Isaac has just finished his first year of graduate study at the University of Oregon.

\section{PRESS SUMMARY}

This paper investigates the affine symmetry tensors, an exciting new generalization of Killing vectors, generators of symmetries, in the Minkowski spacetime of special relativity. While partial results are known about the affine symmetry tensors for valence $1 \& 2$, affine tensors of valence $n>2$ have never before been exhibited. In this article, we discuss a computational algorithm to compute affine tensors in Minkowski spacetime, and discuss the results for affine tensors of valence $2 \leq n \leq 7$. These results are used to make several conjectures about the spaces of affine symmetry tensors analogous to statements about the spaces of Killing tensors. 


\section{APPENDIX}

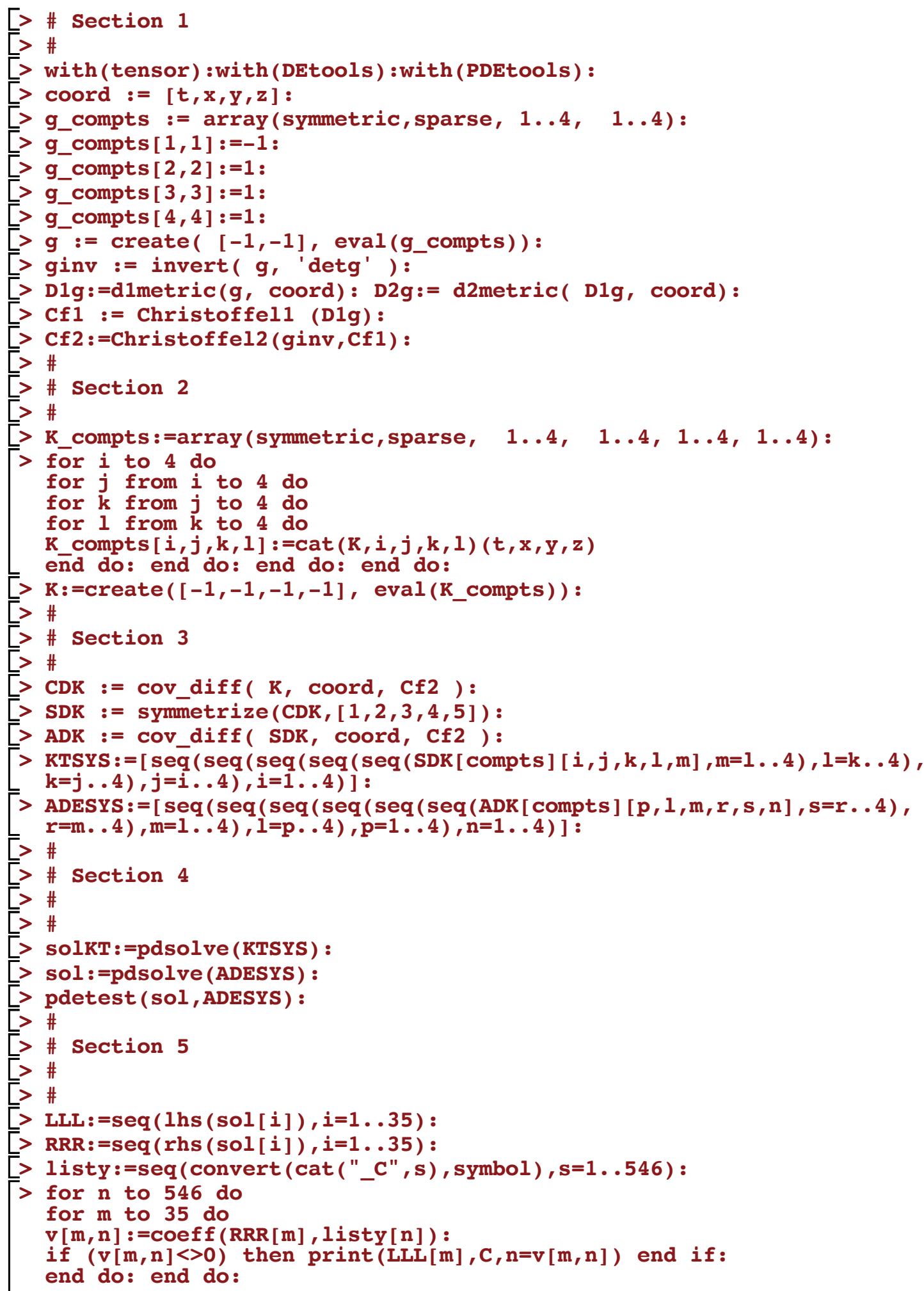

